\title{
WIRELESS MESH NETWORKS CAPACITY IMPROVEMENT USING CBF
}

\author{
Youssef Saadi ${ }^{1}$, Bouchaib Nassereddine ${ }^{2}$, Soufiane Jounaidi ${ }^{3}$ \\ and Abdelkrim Haqiq ${ }^{4}$ \\ ${ }^{1,2,3}$ Computer, Networks, Mobility and Modeling laboratory \\ Department of Mathematics and Computer \\ FST, Hassan 1st University, Settat, Morocco \\ ${ }^{4} \mathrm{e}-\mathrm{NGN}$ Research group, Africa and Middle East
}

\begin{abstract}
Wireless mesh network has recently received a great deal of attention as a promising technology to provide ubiquitous high bandwidth access for a large number of users. Such network may face a significant broadcast traffic that may consequently degrade the network reliability.

In this paper, we have focused interest to wireless mesh network based IEEE 802.11s and we have designed a self-pruning method to control and reduce the broadcast traffic forwarding. Our scheme, namely Control of Broadcast Forwarding (CBF), defines two behaviours to manage the broadcasting operation. Routing packets are managed differently from data broadcast messages to avoid afflicting the routing process.

The simulations results show that CBF ameliorates the network capacity by reducing considerably the number of redundant packets, improving the end to end delay and providing high reachability and packet delivery ration.
\end{abstract}

\section{KEYWORDS}

Flooding Protocols, Wireless Mesh Network, Broadcast Storm

\section{INTRODUCTION}

In multi-hop wireless networks, the trivial way to broadcast a message to all network nodes is via flooding that is a network-wide broadcasting operation by which a packet is disseminated to all other nodes with some nodes selected as relays.

Broadcasting by flooding is an important process used for path and topology discovery in many unicast routing protocols such as AODV, DSR, ZRP ... it is used also by many applications that may include service discovery, address auto configuration or network self-organization.

However such operation is costly and may lead to the broadcast storm problems as referred in [16].

Redundancy, collision and contention are features of the broadcast storm problems that seriously degrade the network performances and hinder the transmission of data packets [5].

Actually, flooding a wireless network based CSMA/CA brings the following drawbacks:

DOI : 10.5121/ijwmn.2015.7301 
- Contention: after receiving the same broadcast message, some neighbors decide to retransmit it at correlated times. Theses transmissions will contend with each other on channel access.

- Redundant retransmissions: a node decides to rebroadcast a message to its neighbors while they have already received the message.

- Collision: the lack of CTS/RTS dialogue, the absence of collision detection and the deficiency backoff mechanism make collision more likely to occur.

These problems become increasingly likely in a wireless mesh network (WMN) based IEEE 802.11s. Actually:

- Flooding is strongly used by HWPM [7] which is the default routing protocol in such network.

- The network control, routing, and topology maintenance rely heavily on layer-2 broadcasting.

- A wireless mesh network may bridge wired networks which deploy many broadcastbased applications such as the address resolution protocol (ARP), the spanning-tree protocol (STP), and Dynamic Host Configuration Protocol (DHCP).

- The WMN ad hoc and infrastructure planes may share the same channel and overlap their coverage area.

These features result in a considerable augmentation of broadcast traffic that can significantly hinder the transmission and routing of unicast data thereby degrading the network reliability.

Previous proposed schemes delay the retransmission of received broadcast packets for a Random Assessment Delay (RAD) which allow nodes sufficient time to receive redundant packets and ascertain whether a rebroadcast is needed or not. The RAD may prevent collisions by differentiating time of retransmissions.

Actually, the RAD deployment may cause serious problems in CSMA/CA based wireless networks. The backoff mechanism is triggered whenever contention occurs by delaying randomly the retransmissions. This delay will be added to the RAD resulting on much more delays that may affect the packet runtime cost.

Previous works does not carry about saving the routing information. They may force using non optimal paths for certain destinations.

Hence, in our approach we address these problems by abandoning the RAD deployment and by processing packets according to their types. Data packets will be manage differently from routing packets.

\section{WIRELESS MESH NETWORKS BASED IEEE 802.11s}

\subsection{Introduction}

Wireless Mesh Networks (WMNs) based IEEE 802.11s [17] are the next step in the evolution of IEEE 802.11 Wireless Local Area Networks (WLANs). They tend to extend the coverage area of WLANs by wireless dynamic association of access points. Unlike WLANs, mesh networks are a particular type of Mobile Ad hoc Networks (MANETs) with low mobility. They are selforganized, self-configured, self-healing and self-discovering. 
Typically, a wireless mesh network based IEEE $802.11 \mathrm{~s}$ is a set of stationary wireless routers (i.e., Mesh points) communicating in multi-hop and forming a kind of backhaul behind access points (i.e., Mesh Access Points) interconnecting client devices (Mesh Stations). The mesh points forward data to or from wireline entry points (i.e., Mesh Portal Points) that are gateways bridging external networks (i.e., Internet).

A WMN is considered hybrid between wireless ad hoc networks and infrastructure-based wireless networks, thus providing flexibility in building and expanding the network [Figure 1]. The infrastructure plane is the area covered by a MAP to serve Mesh stations. The ad hoc plane is the area covered by MPs, MAPs and MPPs to forward the traffic on the infrastructure plane.

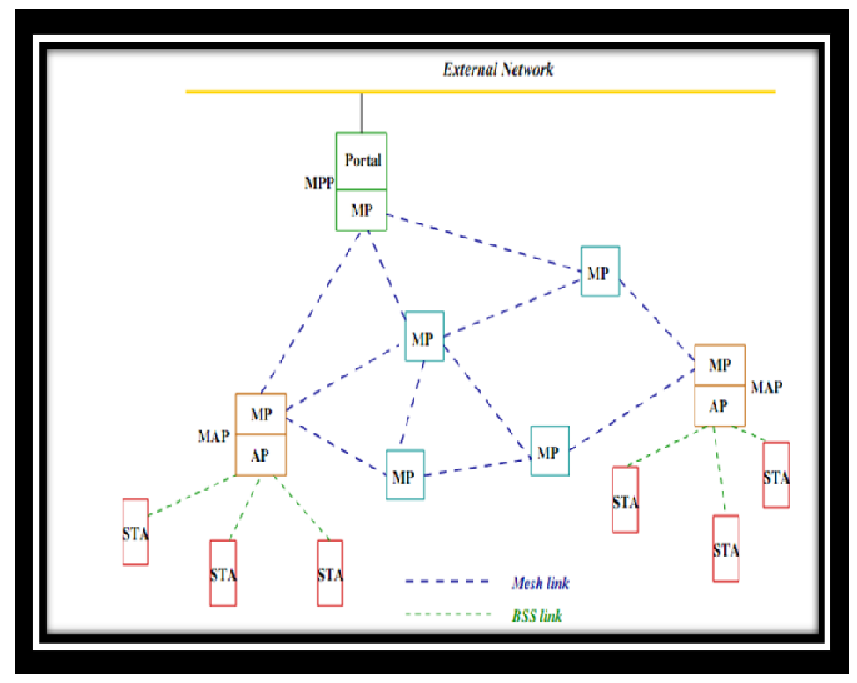

Figure 1. WMN based IEEE 802.11s architecture [17]

\subsection{Routing Procedure}

Routing in WMNs based IEEE 802.11s uses layer 2 addressing instead of IP addresses. The 11s task group has set a target configuration containing up to 32 network entities which can participate in routing. But configurations with more nodes remain possible.

Hybrid Wireless Mesh Network (HWMP) [7] is the default routing protocol used in such networks. It combines between two types of protocols:

The reactive RM- AODV protocol which is a variant of the ad hoc on demand vector protocol [12] using the Air Time metric. In this protocol the route is established on demand. Actually, when a Mesh Point decides to communicate with another one that he does not know the route, it initiates a discovery process to look for an optimized routing path. So, it broadcasts by flooding a path request packet (PREQ) that may cross multi hops to reach the destination or a Mesh Point knowing already a route to this destination. At the other hand, on receiving the PREQ, the destination Mesh Point answers back the source by sending a unicast route reply packet PREP that indicates the reverse path to the destination.

The proactive protocol TBR (Tree Based Routing) most often used for routing to gateways. It configures a particular MP as the root of the tree (e.g., bridge linking an external network). The protocol proceeds in two phases, first phase consisting of tree construction: here the root 
periodically broadcasts a message "Root Announcement" with the distance field set to zero. The MPs then rebroadcast this message while updating the distance to the root. At the end of this phase, each MP has chosen a MP father. This MP father is located on the shortest path to the root. The second phase consists of subscribing the MPs and their stations at the root by sending a "Gratuitous PREP" message. Now when an MP S wants to send a message to an MP D, it sends it to the root, which relays it to the MP D.

In previous works $[14,15]$, we have evaluated by simulations our approach, namely control of broadcast forwarding (CBF). The basic ideas behind were to compute how much CBF can reduce routing broadcast traffic impact on unicast data transmissions and how much rebroadcasts could be saved.

In this work, we enhanced CBF to guarantee more network coverage and more redundant packets decrease. We evaluated our scheme in comparison with Blind Flooding according to several simulation scenarios taking into account various parameters like network density, topology and traffic load.

Reminder of this paper is organized as follows:

Section 3 presents related flooding algorithms. Section 4 describes the Control of Broadcast Forwarding scheme. Section 5 presents the simulation model and shows the obtained results. Section 6 concludes this paper.

\section{RELATED WORKS}

Many works were proposed in the past to mitigate the broadcasting problems in MANETs. They can be categorized into two basic classes.

There are schemes involving the use of neighbourhood knowledge to prune or retransmit the received broadcast message. Two approaches are defined: self-pruning and dominant pruning schemes. In the self-pruning algorithms, each node decides itself to prune the received broadcast message. In dominant pruning approach, only a subset of nodes is selected to forward the broadcast message.

Other protocols try to delay the rebroadcasting operation for a random assessment delay (RAD) in which various observations are performed including neighbours density, number of duplicated packets, and the signal strength. Up on that and according to a predefined threshold, each receiving node may decide to rebroadcast or not the broadcast message.

Below are listed famous broadcasting methods applied for MANETs:

Authors in $[2,9]$ have identified the simple flooding protocol. This scheme requires from each node to retransmit the received broadcast packet for the first time after a small random delay (JITTER). Duplicates are discarded immediately. In a network of $n$ nodes, this results on $n$ retransmissions.

Simple flooding is costly in term of number of retransmitting node, contention and collisions likelihood. These problems are referred as the broadcast storm problems. However it remains a simple protocol for broadcasting and multicasting in non-dense or high mobile ad hoc networks as it is proposed by the IETF Internet Draft [9]. Simple flooding is deployed in HWMP the default routing protocol of IEEE 802.11s wireless mesh networks. 
The authors in [16] have identified the broadcast storm problem through analysis and simulations. They have designed counter-based, probabilistic-based, distant-based, location-based and clusterbased broadcasting schemes to reduce redundancy and collisions in mobile ad hoc networks. The results concluded that counter-based protocol can significantly decrease the number of retransmitting nodes when the network is dense. Location-based scheme can eliminate many redundant packets under all kind of host distributions, but it involves the use of GPS receivers which is costly in mobile ad-hoc networks, particularly in term of energy consumption.

In [3], researchers have defined two flooding methods: self-pruning and dominant pruning which both utilize the neighbourhood information to reduce redundancy. Both schemes perform better than simple flooding, particularly in networks with low mobility rate.

The authors in [4] determined some deficiencies of the dominant pruning method. They proposed two improvements: the total dominant pruning and the partial dominant pruning algorithms. Simulation results demonstrate that both total and partial schemes achieve better performances that the original dominant pruning algorithm specially on reducing the number of redundant packets.

In [10], an efficient broadcast protocol for MANETs (AHPB) is defined. The algorithm chooses a set of nodes, namely Broadcast Relay Gateways (BRGs), which will rebroadcast a flooded packet. The others nodes will not participate in rebroadcast operation. The BRGs constitute a connected dominating set of the network and can achieve a high reliability. AHPB reduces significantly the redundant retransmissions and saves the network bandwidth. It can be applied in static networks to provide efficient broadcast service.

The authors in [11] defined a self-pruning scheme called scalable broadcast algorithm (SBA) that uses 2-hop neighbourhood knowledge to prune redundant rebroadcast. The protocol requires 2hop hello messages exchange to take decision on broadcasting or not the received broadcast packets. Each node whenever it has uncovered neighbours by the sender transmission it schedules a retransmission after a random delay (RAD) in which it learns about covered neighbours from received duplicate packets. After RAD expiration, if it still has uncovered neighbours it will rebroadcast the message otherwise it will discard it.

Williams and T. Camp in [18] have classified the broadcast techniques into several categories and tried to simulate a subset of each category to pinpoint specific features to network conditions like congestion, mobility and density. The results demonstrate that methods using a random access delay (RAD) for rescheduling the retransmissions suffer from congestive networks. Also, it has been observed that the mobility afflicts the neighbours' knowledge methods while probabilisticbased algorithms are disturbed by increasing network density.

In [8] M. Jacobsson designed a self-pruning scheme, namely prioritized flooding with Self Pruning (PFS) which is a combination between counter-based scheme and flooding with selfpruning algorithm. A new design of RAD was adopted based on an estimation of the uncovered neighbors. The scheme requires only one hop hello messages which lead to decreased overhead in comparison with other protocols. PFS performs high reachability and reduce considerably the number redundant packets. However it costs in end to end delay. 


\section{CONTROL OF BROADCAST FORWARDING PRINCIPAL}

CBF approach is a self-pruning protocol using two hop neighbourhood information to decide whether to rebroadcast or eliminate the received broadcast packet for the first time. Duplicates are discarded immediately based on packet sequence number. The two-hop neighbourhood information is obtained by periodically exchanging hello messages. After this exchange, each mesh point builds a table containing the list of its immediate neighbours and their neighbours. An example of such table is shown below in [Figure 2].

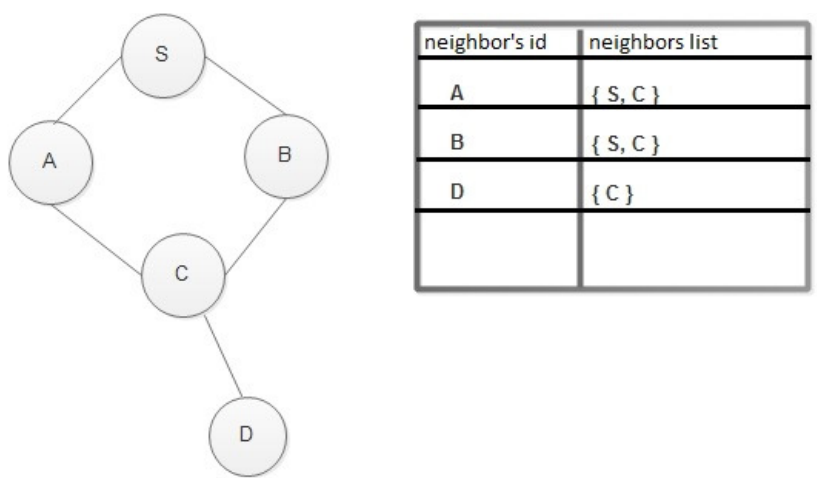

Figure 2. Built neighbours table by node $\mathrm{C}$

CBF manages differently broadcast traffic accordingly to packet type information element. This make CBF more tolerant to routing process.

$\mathrm{CBF}$ uses the following notations and assumptions:

NG (M): the one hop neighbours list of the mesh point $\mathbf{M}$.

Degree $s_{S}(\mathbf{M})$ : the number of uncovered neighbours of mesh point $\mathbf{M}$ by the sender transmission performed by $\mathbf{S}$.

DT $_{\mathbf{S}}(\mathbf{M})$ : the table of degrees which is a map containing pairs of $\mathbf{M}$ 's covered neighbours and their degrees.

$\mathbf{U C}_{\mathbf{S}}(\mathbf{M})$ : the list of uncovered neighbours of the mesh point $\mathbf{M}$ by the sender dissemination performed by node $\mathbf{S}$.

Actually, on receiving a routing broadcast packet, $\mathrm{CBF}$ checks if the receiver's neighbours have been already covered by the broadcast. If so, the routing packet will be discarded, else the packet will be forwarded.

When receiving data broadcast packet, $\mathrm{CBF}$ operates as follows:

On receiving a broadcast message from node $\mathbf{S}$, the receiver $\mathbf{H}$ performs the following procedure:

It checks the sender neighbours list $\mathbf{N G ( S )}$

If NG (S) $-\{\mathbf{H}\}$ includes NG $(\mathbf{H})-\{\mathbf{S}\}$ then $\mathbf{H}$ discards the packet immediately.

Else, meaning that there are uncovered neighbours, $\mathbf{H}$ performs the following actions:

- It builds the degrees table $\mathbf{D T}_{\mathbf{S}} \mathbf{( H )}$

- If it has the maximum degree Degree ${ }_{s}(\mathbf{H})$ among its immediate covered neighbours, then it rebroadcasts the packet. 
- Else $\mathbf{H}$ checks for existing covered mesh points that have max degree and that may cover all its uncovered neighbours. If such nodes exist, the packet will be discarded, else the packet will be retransmitted.

An example of CBF operation is shown in [Figure 3]:

In this example, the mesh point $\mathbf{S}$ broadcasts a message that will be received by its immediate neighbours $\mathbf{A}, \mathbf{B}$ and $\mathbf{C}$.

On checking the sender's neighbours list, A, B and C will know about the existence of uncovered neighbours by $\mathrm{S}$ transmission.

The uncovered neighbours of $A$ are nodes $\{1,2\}$ (i.e., $\mathbf{U C}_{\mathbf{S}}(\mathbf{A})=\{1,2\}$ ), so its degree is 2 (i.e., Degree $\left.\__{S}(A)=2\right)$.

The uncovered neighbours of $\mathbf{B}$ are nodes $\{4,5\}$, so its degree is 2 (i.e., Degree $\_(\mathbf{B})=\mathbf{2}$ ).

The uncovered neighbours of $\mathrm{C}$ are nodes $\{1,2,3,4,5\}$, so its degree is 5 (i.e., Degree $\_(\mathbf{C})=\mathbf{5}$ ).

$\mathrm{C}$ has the maximum degree and its retransmission may cover uncovered neighbours of both $\mathrm{A}$ and B. Thus, $\mathrm{C}$ will rebroadcast the packet while $\mathrm{A}$ and $\mathrm{B}$ will discard it.

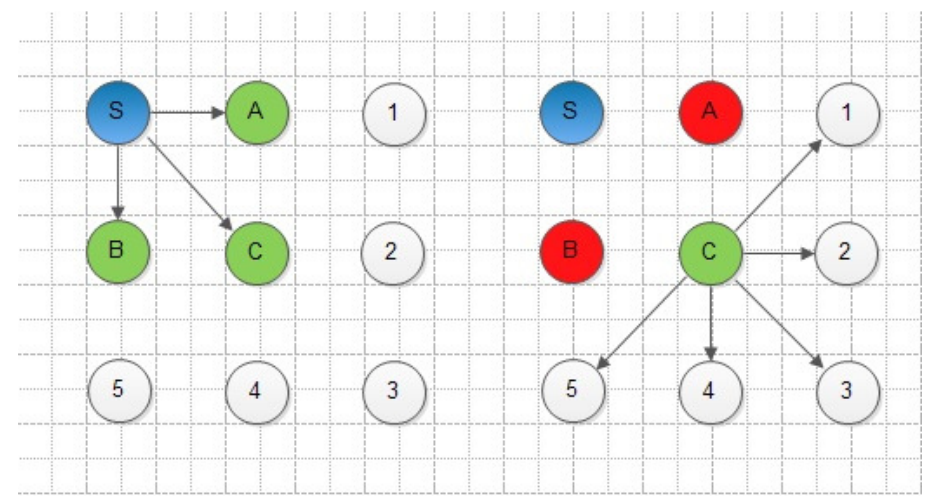

Figure 3. Example of CBF operation

\section{SiMULATION AND RESULTS}

In this section we evaluated our algorithm CBF in comparison with Blind Flooding which is the default scheme used in HWMP routing process and in many other applications involved in Ethernet networks that may be bridged to the mesh. The main focus of the simulations is to observe CBF dealing with broadcast data packet according to various network scenarios. Features like network density and traffic load were studied here.

We used NS-2 [1] to simulate and compare the performance of both protocols. We selected the Distributed Coordination Function (DCF) of IEEE 802.11[6] as the MAC protocol. RTS/CTS/ACK exchange was disabled since we are only concerned by broadcasting operation. In each simulation scenario, flooding messages were generated at random times by random mesh points. Nodes were placed in such a way to form a square grid mesh network as shown in [Fig. 4], 
and they are supposed to be static since we aim to simulate a wireless mesh network where mobility is not a critical constraint. Grid topology is a better choice for wireless mesh networks according to [13]. The table [Table 1] summaries the common parameters used in all simulations.

Table 1. Common parameters.

\begin{tabular}{|c|c|}
\hline Number of nodes (n) & $9-225$ \\
\hline MAC layer & IEEE 802.11 \\
\hline Transmission data rate & $11 \mathrm{Mbits} / \mathrm{s}$ \\
\hline Basic rate & $1 \mathrm{Mbits} / \mathrm{s}$ \\
\hline Flooding message payload & $64 \mathrm{Bytes}$ \\
\hline Flooding rate & 2packets/s $; 10$ packets/s \\
\hline Hello Interval & $1 \mathrm{~s}$ \\
\hline Transmission range & $250 \mathrm{~m}$ \\
\hline Simulation time & $1000 \mathrm{~s}$ \\
\hline
\end{tabular}

To compare the performances of both flooding protocols we used the following parameters:

Reachability: It expresses how many nodes have been reached by the flooding message. For example, if the network size is 50 nodes and a flooding message was received by 40 , so the reachability is $40 / 49=81.63 \%$.

Retransmissions: it is the broadcast cost criteria that determine the number of rebroadcasting nodes for a flooding message.

End to end delay: is the time from the first moment of sending a flooding message until its reception by the last node in the network.

Overhead: is the number of bytes transmitted by unit time. It includes both flooded and hello messages.

\subsection{Non dense WMN}

In this experiment, each node is distant from its closed neighbours by fixed distance that is equal to $175 \mathrm{~m}$. So, each node can communicate directly only with its immediate closed neighbours. To communicate with the others nodes, a multi hop process is needed. The flooding rate is fixed to 2packets/second (i.e., low traffic) and to 10packets/second (i.e., heavy traffic). 
International Journal of Wireless \& Mobile Networks (IJWMN) Vol. 7, No. 3, June 2015

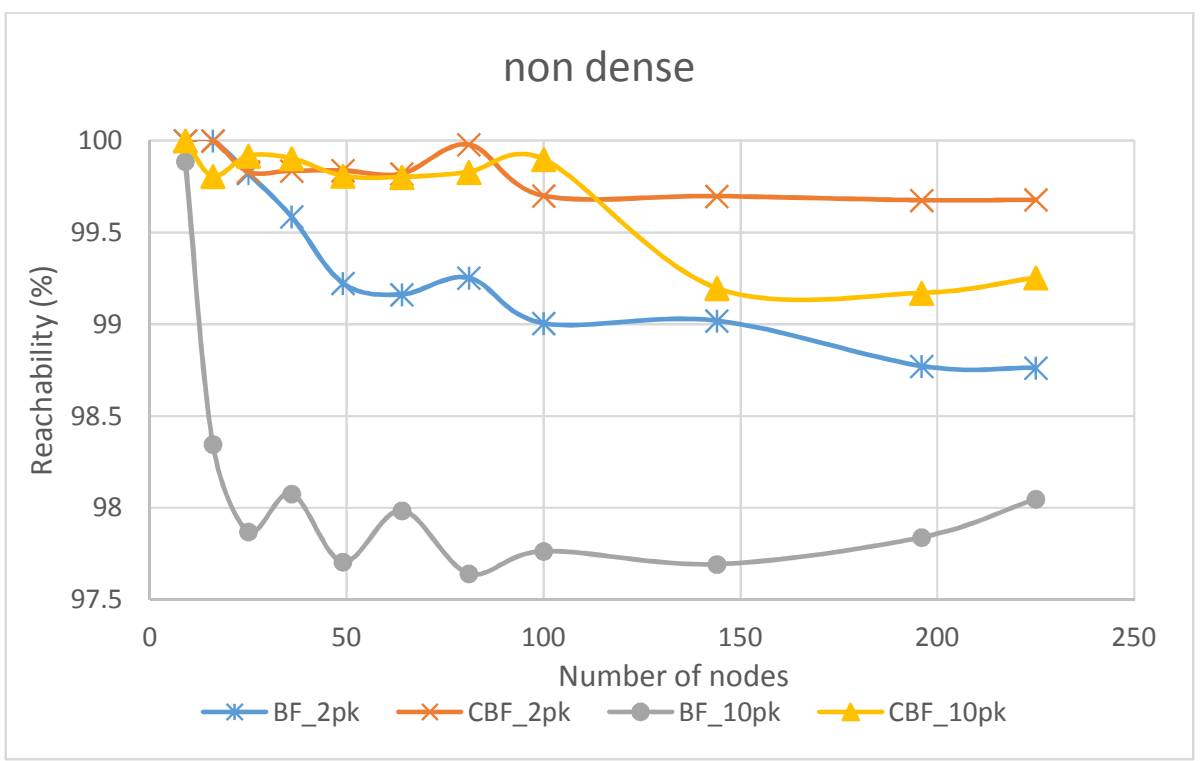

Figure 4. CBF \& BF reachability in non-dense network

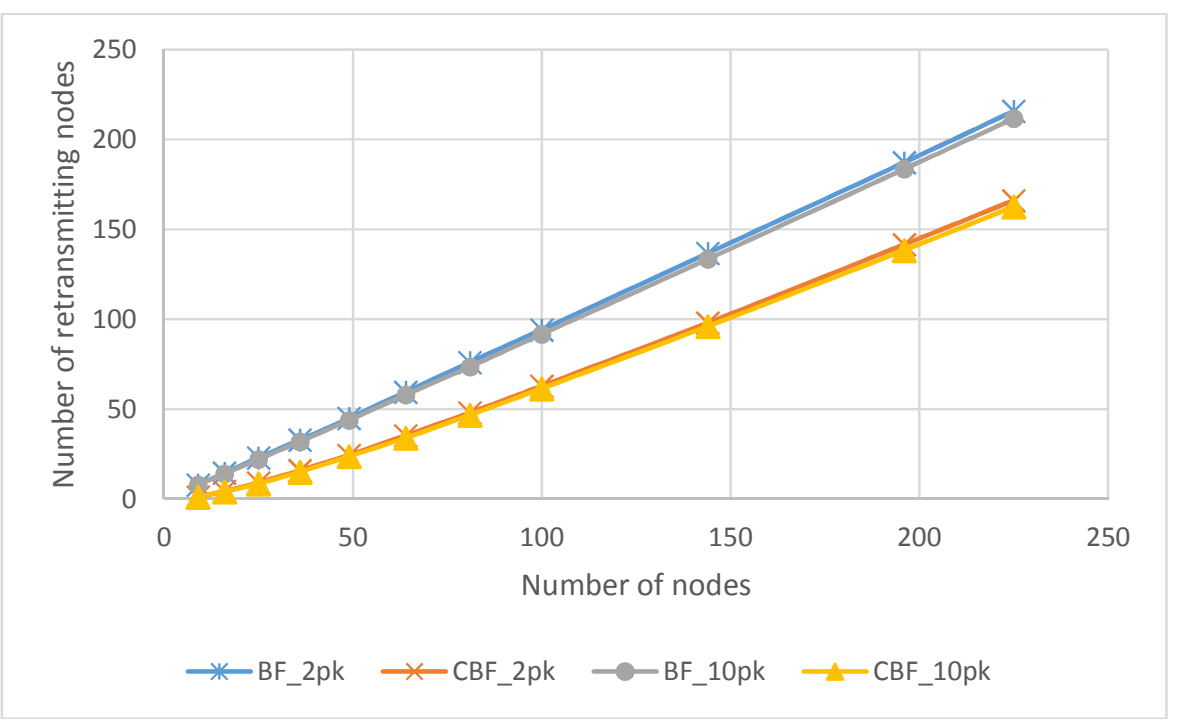

Figure 5. CBF \& BF saved rebroadcasts in non-dense network 
International Journal of Wireless \& Mobile Networks (IJWMN) Vol. 7, No. 3, June 2015

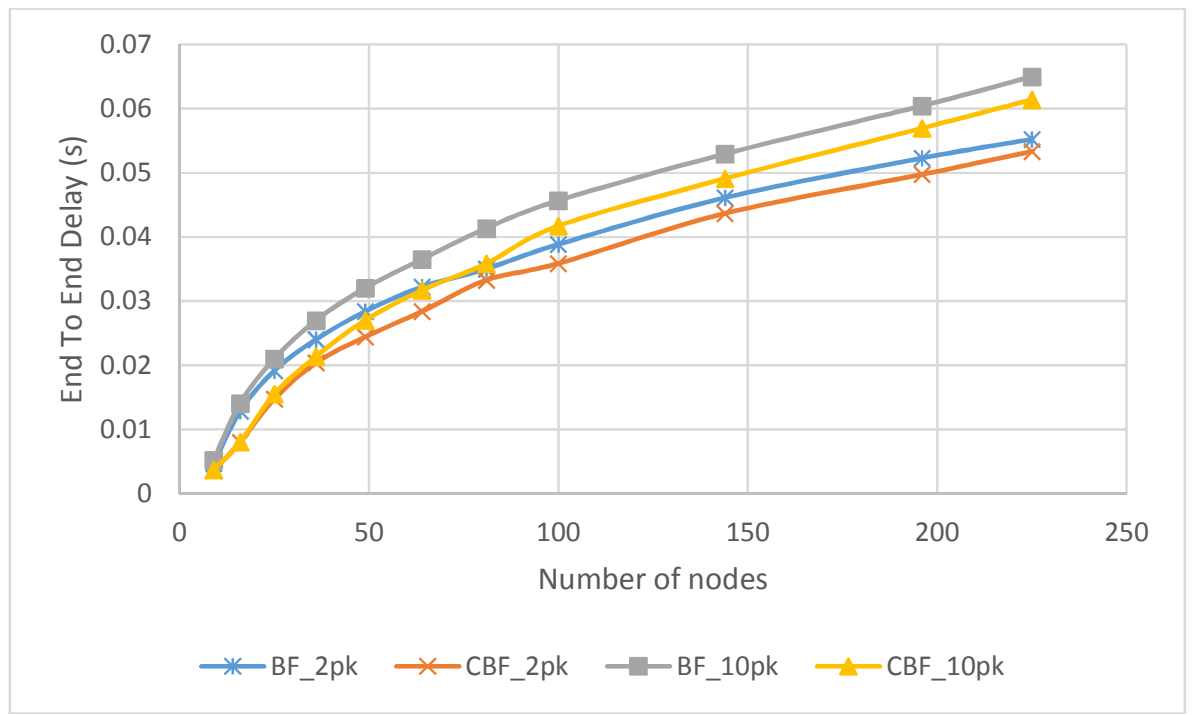

Figure 6. CBF \& BF Average End to End Delay in non-dense network

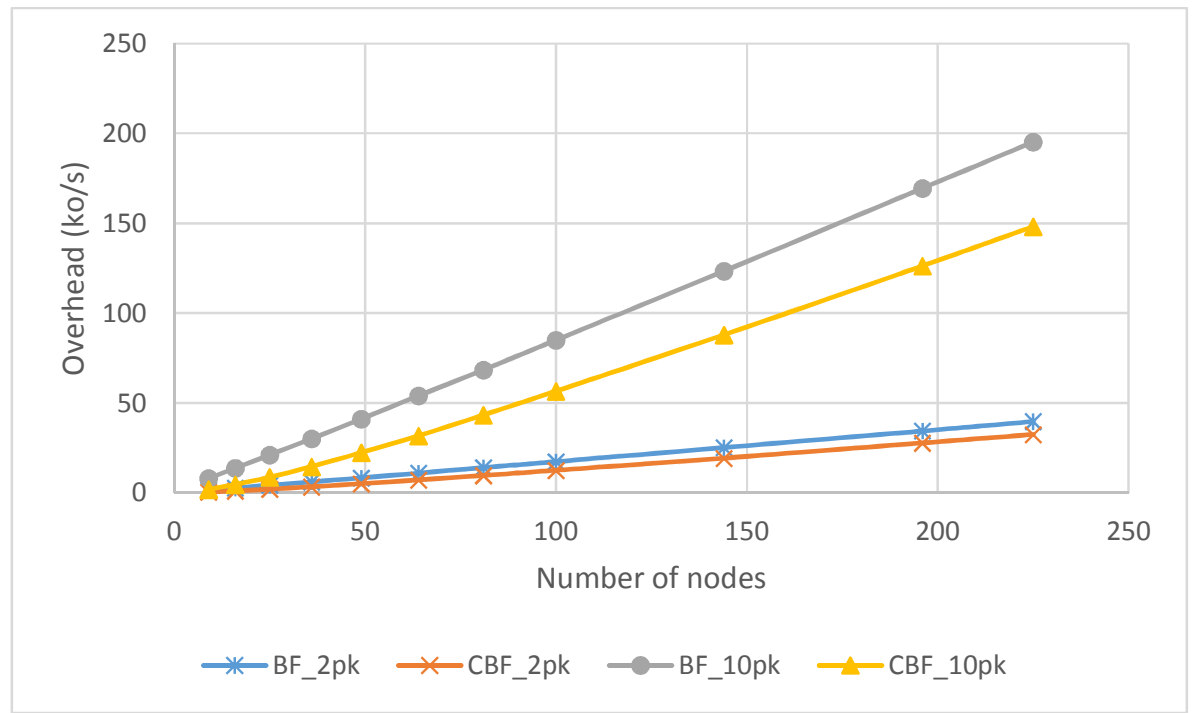

Figure 7. CBF \& BF overhead in non-dense network

The results, presented by figures 4,5,6 and 7, show that CBF ameliorates the WMN capacity by reducing the number of redundant packets, the network overhead and the average end to end delay.

Actually, in the networks with low traffic, CBF produce similar reachability as BF. It performs in average $99.82 \%$ network reachability against $99.32 \%$ provided by BF. When traffic is high, many packets were not received due to contention and collisions resulting of strong demand on 
rebroadcasting at correlated times. This explains why reachability is reduced with BF operation to reach in average $98 \%$ of the network nodes. However CBF keeps a uniform percentage of $99.68 \%$ which is a good value to consider in heavy traffic networks. The good reachability is consequence of the choice of the differentiating time (JITTER value) before rebroadcasting which is favouring nodes with most uncovered neighbors to retransmit first and faster.

The end to end delay is slightly improved by CBF in both low and heavy traffic scenarios. Also, $\mathrm{CBF}$ achieve lower overhead in comparison with BF. We estimate an average overhead reduction of $4 \mathrm{ko} / \mathrm{s}$ in low traffic network and $24 \mathrm{ko} / \mathrm{s}$ in high traffic network.

CBF reduces considerably the number of redundant packets. We estimate $50 \%$ of saved rebroadcasts in both low and heavy traffic network.

\subsection{Dense WMN}

We reduce the distance separating closed neighbours to become $75 \mathrm{~m}$. Same flooding rates are chosen in this scenario.

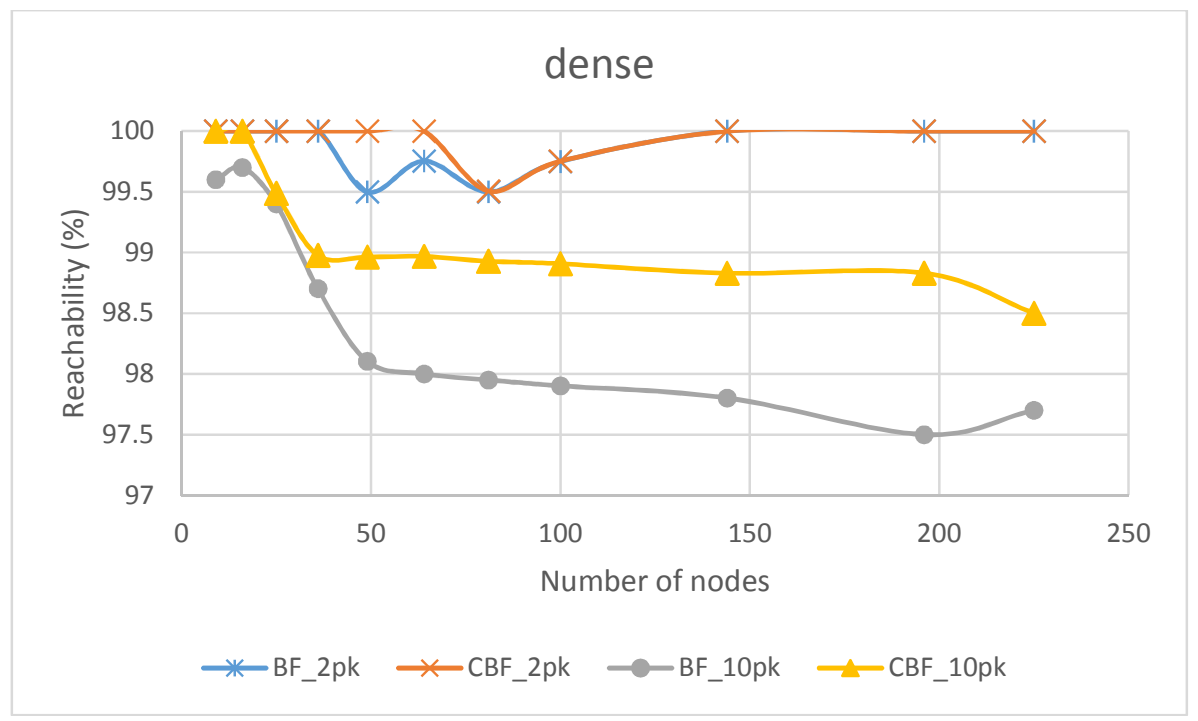

Figure 8. CBF \& BF Reachability in dense network 
International Journal of Wireless \& Mobile Networks (IJWMN) Vol. 7, No. 3, June 2015

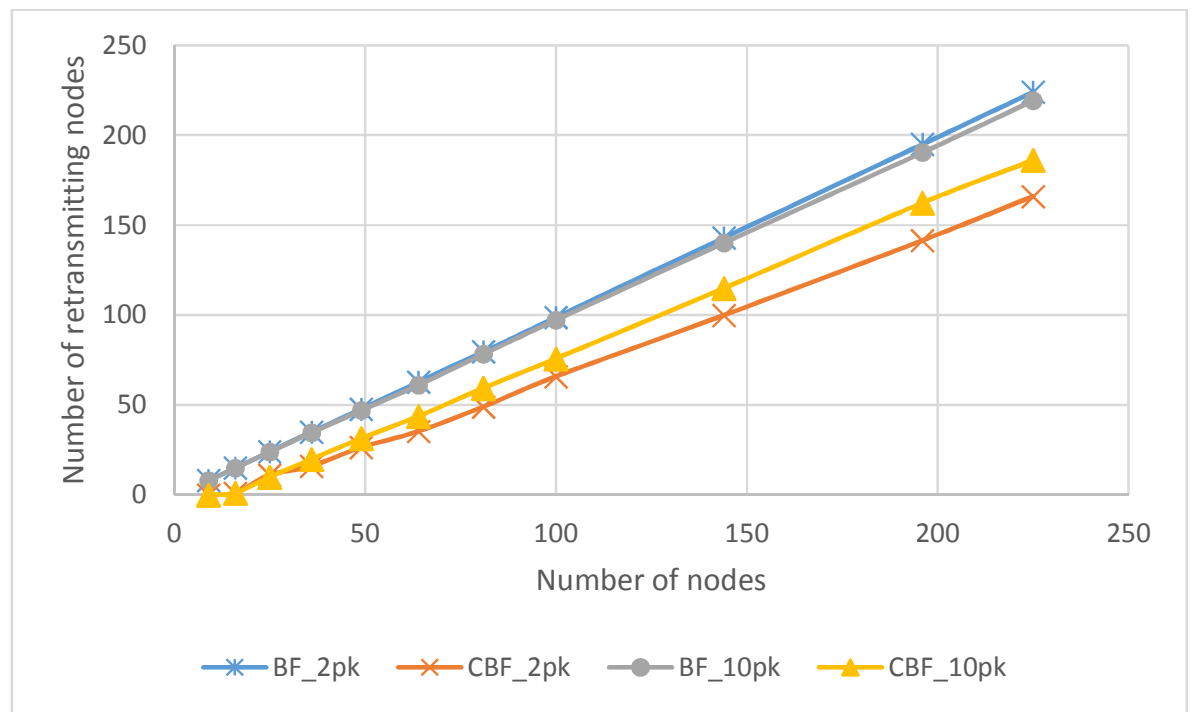

Figure 9. CBF \& BF Saved Rebroadcasts in dense network

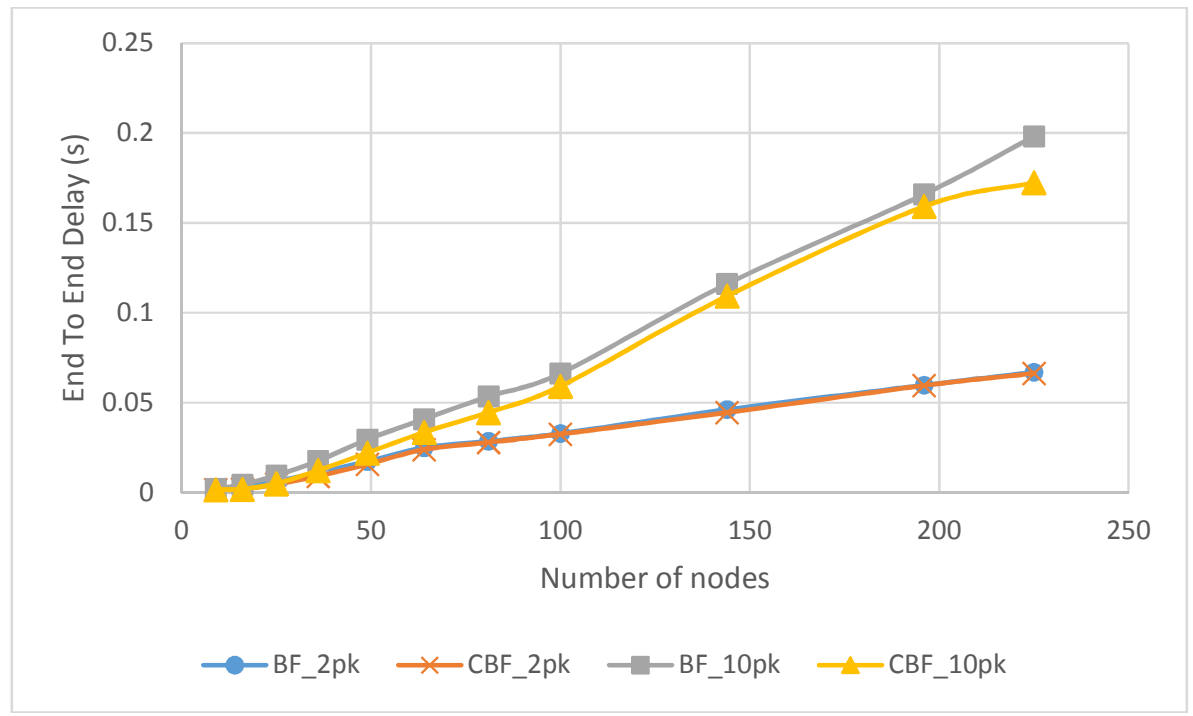

Figure 10. CBF \& BF Average End to End Delay in dense network 


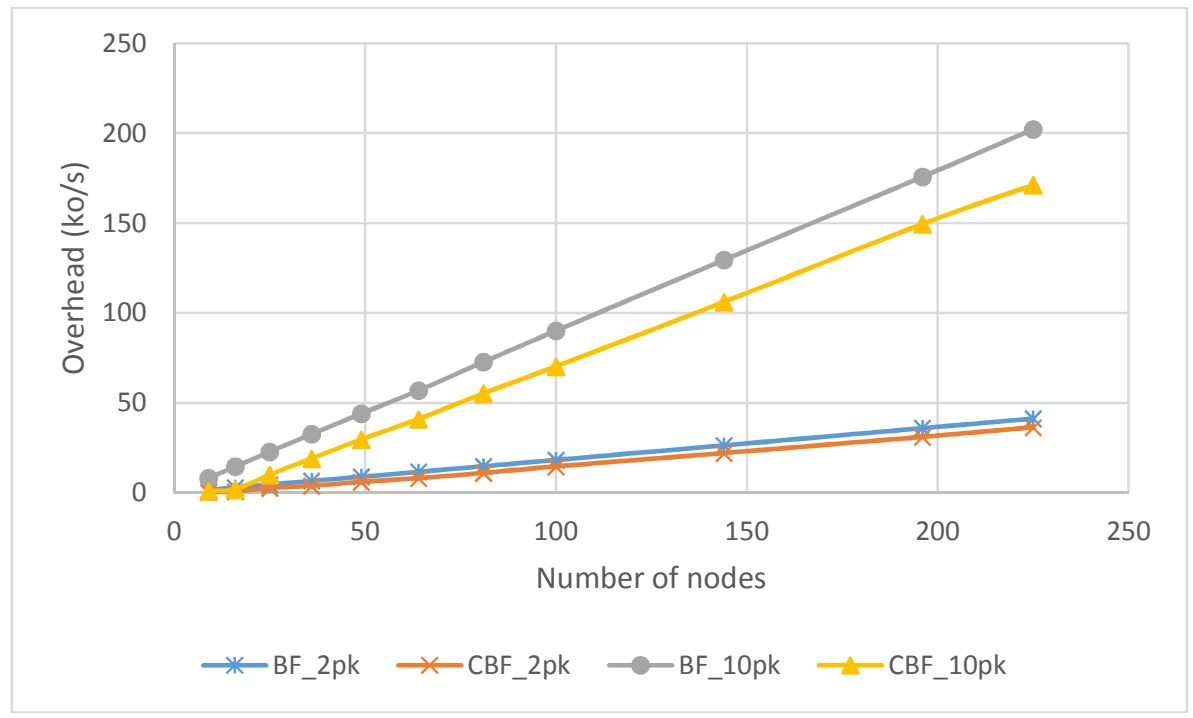

Figure 11. CBF \& BF overhead in dense network

In dense networks, $\mathrm{CBF}$ shows better performances than BF. It performs high reachability [Figure 8 ] in low traffic networks just like BF with an average value of $99.95 \%$. However, we observe a decreased value in high traffic networks for both protocols. The average reachability is estimated to $98 \%$. This can be explained by the non-availability of the complete neighbouring information when needed. A solution to that problem is to adjust the hello message interval to the network size. It must be reduced or increased dynamically according to the network density.

Just like in non-dense networks, CBF achieve better performances in terms of average end to end delay [Figure 10], saved rebroadcasts and overhead.

The saved rebroadcasts [Figure 9] is almost estimated to 50\% in low traffic network and to $42 \%$ in high traffic network. The number of retransmitting nodes is increased when it is about heavy traffic due to non-exact neighbouring information provided by hello messages exchange.

The overhead [Figure 11] is in average reduced about $4 \mathrm{ko} / \mathrm{s}$ in low traffic networks and about $18 \mathrm{ko} / \mathrm{s}$ in high traffic networks.

\section{Conclusion}

Broadcasting by flooding remains a major problem in multi hop networks and particularly in wireless mesh networks. In this study, we evaluated our scheme, namely $\mathrm{CBF}$, in comparison with Blind Flooding protocol. Several experiments were chosen to evaluate the performances of CBF. The results show that CBF behaves well in both dense and non-dense networks and in both low and high traffic networks. The performance parameters were considerably improved, especially, in terms of average end to end delay, overhead, reachability and number of saved rebroadcasts. In future works we will target improving the reachability in high traffic networks to keep it closed to $100 \%$. We will also compare our scheme with the most suitable broadcasting protocols for mesh networks. 
International Journal of Wireless \& Mobile Networks (IJWMN) Vol. 7, No. 3, June 2015

\section{ACKNOWLEDGEMENTS}

The authors would like to thank Martin Jacobsson for his considerable help.

\section{REFERENCES}

[1] K. Fall, K. Varadhan. "The NS Manual” . Vint Project, UC Berkeley, LBL, DARPA, USC/ISI, and Xerox PARC. April 14, 2002.

[2] C. Ho, K. Obraczka, G. Tsudik, K. Viswanath, Flooding for reliable multicast in multihop ad hoc networks, in: Proceedings of the International Workshop on Discrete Algorithms and Methods for Mobile Computing and Communications (DIALM), ACM, 1999, pp. 64-71.

[3] H. Lim and C. Kim, Flooding for Wireless Ad-Hoc Networks, in Computer Communications vol. 24, no. 3-4, pp. 353-363, 2011.

[4] W. Lou, J. Wu, On reducing broadcast redundancy in ad hoc wireless networks IEEE Transactions on Mobile Computing 1 (2002) 111-123.

[5] W. Lou, J. Wu, Toward broadcast reliability in mobile ad hoc networks with double coverage, IEEE Transactions on Mobile Computing 6 (2007) 148-163.

[6] IEEE 802.11 Standard Working Group, Draft Standard for Information Technology Telecommunications and Information Exchange Between Systems - LAN/MAN Specific requirements Part 11: Wireless LAN Medium Access Control (MAC) and Physical Layer (PHY) Specifications, IEEE P802.11-REVma/D9.0, January 2007.

[7] IEEE 802.11s Task Group HWMP Specification doc.: IEEE 802.11-06/1778r1 November 2006.

[8] M. Jacobsson, C. Guo, I.G.M.M. Niemegeers, A flooding protocol for manets with self-pruning and priorited retransmissions, in: International Workshop on Localized Communication and Topology Protocols for Ad hoc Networks (LOCAN), Washington DC, USA, 2005.

[9] J. Jetcheva, Y. Hu, D. Maltz and D. Johnson. A Simple Protocol for Multicast and Broadcast in Mobile Ad hoc Networks. Internet Draft, draft- ietf-manet-simple-mbcast-01.txt, 2001.

[10] Wei Peng, Xicheng Lu, Ahbp: An efficient broadcast protocol for mobile ad hoc networks, Journal of Science and Technology - Beijing, China, 2002.

[11] Wei Peng, Xi-Cheng Wu, On the reducation of broadcast redundancy in mobile ad hoc networks, In The Sixth Annual International Conference on Mobile Computing and Networking (MobiCom 2000), Boston, USA, August 6-11, 2000.

[12] C.E. Perkins, E.M. Belding-Royer, S.R. Das, Ad hoc on-demand distance vector (aodv) routing, IETF RFC3561, July 2003.

[13] J. Robinson, E. Knightly, A performance study of deployment factors in wireless mesh networks, in: Proceedings of the IEEE International Conference on Computer Communications (INFOCOM '07), 2007, pp. 2054-2062.

[14] Saadi, Y.; Nassereddine, B.; Bennani, S.; Maach, A., "An adaptive approach to control broadcast traffic in wireless mesh networks based IEEE 802.11s," Complex Systems (ICCS), 2012 International Conference on , vol., no., pp.1,7, 5-6 Nov. 2012 doi: 10.1109/ICoCS.2012.6458512

[15] Saadi, Y.; Nassereddine, B.; Haqiq, A., "CBF evaluation through simulation," Next Generation Networks and Services (NGNS), 2014 Fifth International Conference on , vol., no., pp.182,186, 28-30 May 2014 doi: 10.1109/NGNS.2014.6990250

[16] Y.-C. Tseng, S.-Y. Ni, Y.-S. Chen, J.-P. Sheu, The broadcast storm problem in a mobile ad hoc network, Wireless Networks 8 (2/3) (2002) 153-167.

[17] X. Wang, and A.O. Lim, IEEE 802.11s wireless mesh networks: Framework and challenges, Ad Hoc Networks 6 (2008) 970-984.

[18] Brad Williams, Tracy Camp, Comparison of broadcasting techniques for mobile ad hoc networks, In The Third ACM International Symposium on Mobile Ad Hoc Networking and Computing (MobiHoc 2002), Lausanne, Switzerland, June 9-11, 2002. 


\section{Author}

Youssef SAADI received the B.Sc. degree in Computer Engineering in 2005 and M.Sc. degree in Systems and Networks in 2008 from Hassan 1st University, Faculty of Sciences and Techniques of Settat (FSTS), Morocco. He has been working as professor of Computer Sciences in high school since 2006, Berrechid, Morocco. Currently, he is working toward his Ph.D. at FSTS. His current research interests are: Wireless Mesh Network Capacity Amelioration, Flooding Operation Improvement, and Study of Mobile Ad hoc Networks.

Dr. Bouchaib NASSEREDDINE is a professor at Faculty of Sciences and Techniques (FSTS), Hassan $1^{\text {st }}$ University, Settat, Morocco. He is an active member of research team RIME at MOHAMMADIA School of Engineers, Rabat, Morocco and he is a researcher in the Computer, Networks, Mobility, and Modelling Laboratory, at FSTS.

Dr. Abdelkrim HAQIQ has a High Study Degree (DES) and a PhD (Doctorat d'Etat), both in Applied Mathematics, option modeling and performance evaluation of computer communication networks, from the University of Mohamed V, Agdal, Faculty of Sciences, Rabat, Morocco. Since September 1995 he has been working as a Professor at the department of Mathematics and Computer at the Faculty of Sciences and Techniques, Settat, Morocco. He is the Director of Computer, Networks, Mobility and Modeling laboratory and the Coordinator of the education of Computer Engineering at the Faculty of Sciences and Techniques, Settat. He is also a General Secretary of e-Next Generation Networks Research Group, Moroccan section. 